ANNALS OF

CLINICAL

NEUROPHYSIOLOGY

\title{
Usefulness of infrared thermography in diagnosing and evaluating severity of carpal tunnel syndrome
}

Jiwon Yang, Yeong-Bae Lee, Young-Hee Sung, Dong-Jin Shin, Yong-Jin Kim, Hyeon-Mi Park

Department of Neurology, Gil Medical Center, Gachon University College of Medicine, Incheon, Korea

Received: June 9, 2021

Revised: August 5, 2021

Accepted: August 10, 2021

\section{Correspondence to}

Hyeon-Mi Park

Department of Neurology, Gil Medical Center, Gachon University College of Medicine, 21 Namdong-daero 774beon-gil, Namdong-gu, Incheon 21565, Korea

Tel: +82-32-460-3346

Fax: +82-32-460-3344

E-mail:neurohm@gilhospital.com

ORCID

Jiwon Yang

https://orcid.org/0000-0003-1379-6899

Yeong-Bae Lee

https://orcid.org/0000-0001-5952-1423

Young-Hee Sung

https://orcid.org/0000-0002-2840-1338

Dong-Jin Shin

https://orcid.org/0000-0002-4390-2843

Yong-Jin Kim

https://orcid.org/0000-0003-2963-6814

Hyeon-Mi Park

https://orcid.org/0000-0002-7816-9653

Background: Pain and autonomic dysfunction are prominent symptoms in some patients with carpal tunnel syndrome (CTS). Infrared thermography (IRT) has been used to evaluate CTS by measuring the cutaneous temperature and sympathetic vasomotor function.

Methods: This study enrolled the 66 hands of 33 subjects, some of which had clinical CTS and the others were healthy. The enrolled patients completed the Boston Carpal Tunnel Questionnaire (BCTQ) and Historical-Objective scale, and underwent nerve conduction studies (NCSS) and IRT. Skin temperature was measured at the fingertips and the thenar and hypothenar regions in each hand. We analyzed (1) the correlations between self-reported severity, physician-assessed severity, and test results, and (2) the sensitivity and specificity of IRT in diagnosing CTS.

Results: No significant correlation was observed between the results of the BCTQ, NCS, and IRT. IRT had a low sensitivity and high specificity in diagnosing CTS.

Conclusions: IRT cannot replace NCS in diagnosing CTS, nor did it provide an advantage in combination with NCS. However, lower temperatures at the median nerve in some hands with moderate-to-severe CTS suggested the involvement of sympathetic nerve fiber function. Follow-up studies with a larger-scale and complementary design are required to elucidate the relationships.

Key words: Thermography; Nerve conduction; Carpal tunnel syndrome

\section{INTRODUCTION}

Carpal tunnel syndrome (CTS) is one of the most common entrapment neuropathies that clinical electrophysiologists encounter in their practices. Typical symptoms, signs, and findings of demyelination across the wrist in median nerve conduction studies (NCSs) have been the gold standard for diagnosing CTS, since NCSs have had high sensitivity

This is an Open Access article distributed under the terms of the Creative Commons Attribution Non-Commercial License /http:// creativecommons.org/licenses/by-nc/4.0) which permits unrestricted non-commercial use, distribution, and reproduction in any medium, provided the original work is properly cited. 
and specificity (> 90\%) in numerous studies. Nevertheless, reportedly $10-20 \%$ of patients with clinically defined CTS have false-negative NCS results. ${ }^{2}$ The clinical-electrophysiological discrepancy, which is the weak correlation between NCS findings and the severity of CTS symptoms, has also been widely discussed. ${ }^{3-5}$ The main limitation of NCS is that it examines only large myelinated Aß-fibers, and not small myelinated $A \delta$-fibers or unmyelinated $C$ fibers that mediate pain and paresthesia, which might explain this discrepancy. ${ }^{6}$ Infrared thermography (IRT) is a modality for measuring the sympathetic vasomotor nerve function that is mediated by small myelinated and unmyelinated nerve fibers, and it has been used in the fields of pain research and medicine. ${ }^{7}$ The present study evaluated whether IRT could be useful for diagnosing CTS as an ancillary method to NCS, especially in the presence of severe sensory symptoms and mild NCS abnormalities.

\section{MATERIALS AND METHODS}

\section{Subjects}

Patients with CTS who were admitted to the Neurology Outpatient Department of our hospital between March 2018 and January 2019 were recruited consecutively. Eligible patients were initially screened by one of the authors based on detailed history-taking and careful neurological examinations. The inclusion criteria were as follows: 1) the presence of numbness, tingling, or pain in at least two fingers from the thumb (digit 1 [D1]) to digit 4 (D4); 2) symptoms worsening at night (sleep) or during hand movements; 3) clumsiness, weakness, or muscle atrophy in the thenar region; 4) presence of unilateral hand symptom or much worse hand in bilateral symptomatic hands, 5) age $>20$ years, and 6) symptoms lasting for $>2$ months, even if they occurred intermittently. Provocative maneuvers (Tinel's and Phalen's signs) were recorded in all patients, but they were not required for a patient to be enrolled. The exclusion criteria were as follows: 1) the presence of a medical illness that can contribute to CTS (e.g., diabetes mellitus, thyroid disease, chronic liver or kidney disease, or alcohol abuse), 2) the presence of another peripheral nerve disorder (e.g., polyneuropathy, plexopathy, or radiculopathy), 3) previous injury or surgery of the symptomatic hand, 4) the presence of peripheral vascular disorders, or 5) pregnancy or breastfeeding. Written informed consent was obtained from all patients, and all of them underwent NCSs. The control group consisted of healthy subjects without evidence of clinical or electrophysiological CTS. Note that the hands of the enrolled patients that had never experienced any sensory problems and the hands with normal NCS results served as controls along with the included healthy subjects. This study was approved by the local ethics committee of our institution (approval no. GDIRB2017-360).

\section{Assessment of clinical severity}

All patients completed the following patient- and physician-based questionnaires to assess their clinical severity: the symptom severity scale (SSS) and functional status scale (FSS) of the Boston Carpal Tunnel Questionnaire ${ }^{8}$ and the Historical-Objective (Hi-Ob) scale ${ }^{9}$. The SSS and FSS consisted of 11 and 8 items, respectively, and were rated from 1 to 5 points, with higher scores indicating greater pain and disability. The SSS and FSS scores were calculated as the unweighted mean of all answered items for the corresponding scale; that is, the sum score for the responses divided by the number of items. The maximum and minimum scores on the SSS and FSS were 5 and 1, respectively. The Hi-Ob scale was assessed by the same trained neurologist. If the patients had bilateral CTS symptoms, they were instructed to complete questionnaires for each hand. Both the patient and the examining physician were blinded to the NCS results.

\section{NCSs}

NCSs were performed using standard techniques of surface electrical stimulation and recording and an electromyography (EMG) system (Nicolet Synergy, Natus, Middleton, WI, USA). Skin temperature was maintained at above $31.0^{\circ} \mathrm{C}$. Motor, sensory, and mixed NCSs of the bilateral median and ulnar nerves were performed to reveal the possibility of polyneuropathy and asymptomatic CTS. Needle EMG examinations were performed of the median nerve and the muscles innervated by the $\mathrm{C} 6$ and $\mathrm{C} 7$ roots, depending on patient tolerance. For the median motor NCS, compound muscle action potentials (CMAPs) were recorded in the abductor pollicis brevis muscle for stimulation at approximately $5 \mathrm{~cm}$ from the wrist. Normal values were defined as $\geq 5 \mathrm{mV}$ for CMAP amplitude and $\leq 3.9$ ms for distal motor latency (DML). 
For the median sensory NCS, sensory nerve action potentials (SNAPs) were recorded at the wrist with stimulation 1) at the palm (8-9 cm from the recording electrode) and 2) at the index finger using the orthodromic method. The sensory nerve conduction velocity (SNCV) was determined from the peak latency and calculated in meters per second. Normal values were defined as $\geq 10 \mu \mathrm{V}$ for the SNAP amplitude, $\geq 34 \mathrm{~m} / \mathrm{s}$ for the palm-wrist segment, and $\geq 40 \mathrm{~m} / \mathrm{s}$ for the index finger-wrist segment. Electrophysiological CTS was diagnosed according to the guidelines of the American Association of Neuromuscular and Electrodiagnostic Medicine. ${ }^{10}$ Median-ulnar sensory conduction was compared between the wrist and the ring finger, and considered abnormal when the latency difference was $\geq 0.5 \mathrm{~ms}$ if conventional median sensory conduction was normal in both segments. ${ }^{11}$ The NCS results were graded according to the Padua scale for severity on each hand. ${ }^{12}$

Because the numbers of scores on the Hi-Ob and Padua scales differed and the group with moderate CTS was much larger than the other groups (Table 1), we categorized those scores into two and three groups, respectively: 1) the Hi-Ob scale was categorized into class 1 (stages 1 and 2) for paresthesia and class 2 (stages 3, 4, and 5) for objective sensory or motor deficits, and 2) the Padua scale was categorized into class 1 (grades 1 and 2), class 2 (grade 3), and class 3 (grades 4 and 5) depending on whether NCSs revealed abnormal SNCV only, abnormal DML, or abnormal SNAP and CMAP amplitudes, respectively.

\section{IRT}

The skin temperature of both hands was measured using a digital infrared thermographic imaging system (IRIS-XP, Medicore, Hanam, Korea) in an air-conditioned, window-less laboratory room that was maintained at $20-22^{\circ} \mathrm{C}$ and a humidity of $50-60 \%$. The patients underwent IRT at least 24 hours after the NCS. They were prepared for IRT at 24 hours before the examination, with physical therapy, acupuncture, analgesics, and any topical agents affecting the skin not allowed during that time. On the day of the test, the patients were instructed to remove their top and remain in the room for 15 minutes to equilibrate. The temperatures at 14 sites (bilateral D1 to digit 5 [D5] and the bilateral thenar [Th] and hypothenar $[\mathrm{Ht}]$ regions) were measured as the regions of interest (ROIs) (Fig. 1). Because we included patients with
Table 1. Clinical data of the enrolled patients with CTS and healthy controls

\begin{tabular}{|c|c|c|}
\hline & $\begin{array}{l}\text { Patients with CTS } \\
\text { (25 patients, } \\
50 \text { hands) }\end{array}$ & $\begin{array}{l}\text { Healthy controls } \\
\text { (8 subjects, } \\
16 \text { hands) }\end{array}$ \\
\hline Age (years) & $54.2 \pm 12.9(27-78)$ & $38.8 \pm 9.05(27-52)$ \\
\hline Sex (female) & $21(84)$ & $4(50)$ \\
\hline Body mass index $\left(\mathrm{kg} / \mathrm{m}^{2}\right)$ & $25.3 \pm 3.7(19.2-35.6)$ & $20.4 \pm 3.3(16.6-25.1)$ \\
\hline Clinically affected hand & \multicolumn{2}{|l|}{35} \\
\hline Right (unilateral) & \multicolumn{2}{|c|}{$17(34)$} \\
\hline Left (unilateral) & \multicolumn{2}{|c|}{$8(16)$} \\
\hline Bilateral & \multicolumn{2}{|c|}{$5(20)$} \\
\hline $\begin{array}{l}\text { Clinically unaffected } \\
\text { hand }\end{array}$ & \multicolumn{2}{|l|}{15} \\
\hline Abnormal NCS hand ${ }^{a}$ & \multicolumn{2}{|l|}{40} \\
\hline Right (unilateral) & \multicolumn{2}{|c|}{$8(16)$} \\
\hline Left (unilateral) & \multicolumn{2}{|c|}{$2(4)$} \\
\hline Bilateral & \multicolumn{2}{|c|}{$15(60)$} \\
\hline Normal NCS hand & \multicolumn{2}{|l|}{10} \\
\hline $\begin{array}{l}\text { Symptom duration } \\
\text { (months) }\end{array}$ & \multicolumn{2}{|c|}{$16.9 \pm 20.0(2-60)$} \\
\hline $\begin{array}{l}\text { BCTQ-SSS } \\
\text { (in symptomatic hands) }\end{array}$ & \multicolumn{2}{|c|}{$2.98 \pm 1.16(1.27-4.73)$} \\
\hline $\begin{array}{l}\text { BCTQ-FSS } \\
\text { (in symptomatic hands) }\end{array}$ & \multicolumn{2}{|c|}{$2.21 \pm 0.94(1.0-4.63)$} \\
\hline Hi-Ob scale & \multicolumn{2}{|c|}{ Among clinically affected 35 hands } \\
\hline Stage 1 & \multicolumn{2}{|c|}{$6(17)$} \\
\hline Stage 2 & \multicolumn{2}{|c|}{$14(40)$} \\
\hline Stage 3 & \multicolumn{2}{|c|}{$7(20)$} \\
\hline Stage 4 & \multicolumn{2}{|c|}{$8(23)$} \\
\hline Stage 5 & \multicolumn{2}{|l|}{0} \\
\hline Padua's scale & \multicolumn{2}{|c|}{ Among abnormal NCS 40 hands } \\
\hline Grade 1 & \multicolumn{2}{|c|}{$3(7.5)$} \\
\hline Grade 2 & \multicolumn{2}{|c|}{$8(20.0)$} \\
\hline Grade 3 & \multicolumn{2}{|c|}{$21(52.5)$} \\
\hline Grade 4 & \multicolumn{2}{|c|}{$1(2.5)$} \\
\hline Grade 5 & \multicolumn{2}{|c|}{$7(17.5)$} \\
\hline
\end{tabular}

Values are presented as mean \pm standard deviation (range) or number (\%). CTS, carpal tunnel syndrome; NCS, nerve conduction study; BCTQ, Boston Carpal Tunnel Questionnaire; SSS, symptomatic severity scale; FSS, function status scale; Hi-Ob scale, historical-objective scale.

a Abnormal NCS results, regardless of symptoms. 
bilateral CTS with a profoundly symptomatic single hand, we calculated the absolute temperature differences ( $\Delta T$-values) between each two of the seven sites in one hand rather than the thermal asymmetry between the left and right corresponding sites.

With reference to a previous study, ${ }^{13}$ we determined D1, D2, D3, and Th as the locations of median nerve vasomotor innervation, and D5 and $\mathrm{Ht}$ as the location of ulnar nerve vasomotor innervation. D4 was excluded from the analysis for clarity since both median and ulnar nerve innervated in D4. For sensitivity and specificity calculations, a significant difference was regarded as being due to the carpal tunnel lesion when one of $\Delta T(D 1-D 5), \Delta T(D 2-D 5), \Delta T$ (D3-D5), $\Delta T$ (Th- $\mathrm{Ht}$ ) exceeded the reference value and simultaneously $\Delta T$ (D5-Ht) was below the reference value $\left(0.4^{\circ} \mathrm{C}^{14}, 0.6^{\circ} \mathrm{C}^{15}\right.$, and $1.0^{\circ} \mathrm{C}^{16}$, respectively). The median index (MI) was calculated as (D1-D2) + (D2-D3) + (D1-D3), ${ }_{1}^{17}$ and the median-ulnar difference (MU) was calculated as (D1-D5) + (D2-D5) + (D3$\mathrm{D} 5)+(\mathrm{Th}-\mathrm{Ht}){ }^{18}$ Higher $\mathrm{Ml}$ and $\mathrm{MU}$ values mean that the skin temperature in the median nerve distribution area is more variable, and this might reflect a malfunction in sympathetic vasoregulation.

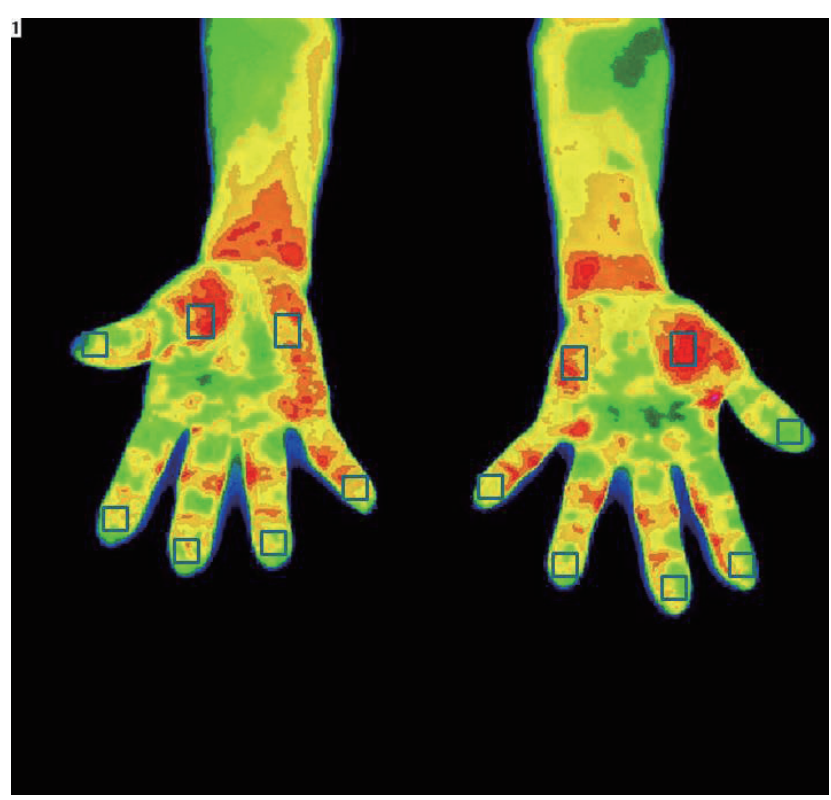

Fig. 1. An example of digital infrared thermographic imaging in a patient with carpal tunnel syndrome. Regions of interest are indicated as squares where the temperature was measured.

\section{Statistical analysis}

Nonparametric tests were performed because Kolmogorov-Smirnov tests indicated that not all of the data conformed to a normal distribution. Continuous variables were examined using Mann-Whitney, Kruskal-Wallis, and univariate linear regression tests, as appropriate. $p$-values of less than 0.05 were considered to indicate statistical significance, and Bonferroni correction was applied for multiple comparisons. The sensitivity and specificity of NCSs, IRT, and their combination in diagnosing clinical CTS were calculated. Correlations between the SSS, FSS, Hi-Ob scale, Padua scale, and IRT results were examined using Spearman's test. All statistical analyses were performed using Statistical Package for the Social Sciences (version 21.0, IBM Corp., Armonk, NY, USA).

\section{RESULTS}

Twenty-five patients with CTS and eight healthy control subjects were enrolled, and all of them underwent neurological examinations, NCSs, and IRT, and completed the study questionnaires (Table 1). The patients with CTS included 21 females (84\%) and 5 patients with bilateral CTS symptoms (20\%). Fifty hands of 25 patients with CTS were evaluated, among which 35 had clinical CTS and 40 had abnormal NCS results. It was particularly interesting that a discrepancy

Table 2. Sensitivity and specificity of thermography and NCS

\begin{tabular}{lcr}
\hline & Sensitivity & Specificity \\
\hline $\begin{array}{l}\text { Regarding } 39 \text { hands with } \\
\text { consistent symptoms (CTS or } \\
\text { normal) and NCS }\end{array}$ & & \\
$0.4^{\circ} \mathrm{C}$ & $13 / 32(40.6)$ & $2 / 7(71.4)$ \\
$0.6^{\circ} \mathrm{C}$ & $6 / 32(18.8)$ & $1 / 7(85.7)$ \\
$1.0^{\circ} \mathrm{C}$ & $4 / 32(12.5)$ & $1 / 7(85.7)$ \\
Based on a pure clinical & & \\
diagnosis of CTS in 50 hands & & \\
Only NCS & $32 / 35(91.4)$ & $8 / 15(46.6)$ \\
NCS or $0.4^{\circ} \mathrm{C}$ & $33 / 35(94.3)$ & $10 / 15(33.3)$ \\
NCS or $0.6^{\circ} \mathrm{C}$ & $33 / 35(94.3)$ & $9 / 15(40.0)$ \\
NCS or $1.0^{\circ} \mathrm{C}$ & $33 / 35(94.3)$ & $8 / 15(46.6)$ \\
\hline
\end{tabular}

Values are presented as number (\%).

NCS, nerve conduction study; CTS, carpal tunnel syndrome. 
between the presence of symptoms and NCS results was observed in our data, as in previous studies: clinical CTS symptoms with normal NCS findings were observed in three hands, and NCS abnormalities without CTS symptoms were observed in eight hands. Patients with CTS tended to have a higher body mass index (BMI).

\section{IRT and NCS}

A side-to-side comparison of hand temperatures at the seven ROls in eight healthy controls showed similarity and high correlation coefficients for each comparison, confirming the presence of symmetry in hand temperatures (Supplementary Table 1). When comparing healthy controls $(n=8)$ and patients with CTS who showed clinical symptoms in total agreement with NCS results $(n=14)$, absolute side-to-side differences in the D2 temperature tended to be greater in patients with $\mathrm{CTS}$ (mean value, $0.71^{\circ} \mathrm{C}$ vs. $0.26^{\circ} \mathrm{C}$ ), though the difference did not reach statistical significance $(p=0.266)$ after adjusting for age, sex, and BMI. Considering all hands ( $n=66$ ), regardless of NCS results, comparisons of regional $\Delta T$ in the same hand between symptomatic CTS and healthy hands did not reveal any differences. However, regardless of symptoms, hands with abnormal NCS results showed higher MI than hands with normal NCS results (mean value, $0.86^{\circ} \mathrm{C}$ vs. $0.54^{\circ} \mathrm{C}$ ), but this difference also did not reach statistical significance $(p=0.055)$ after adjusting for age, sex, and BMI (Supplementary Table 2).

Based on a pure clinical diagnosis of symptomatic and asymptomatic hands, the sensitivity of IRT decreased and its specificity increased as the cutoff value increased (Table 2). The sensitivity and specificity of IRT were not superior to those of NCS. Combining the existence of either IRT (cutoff

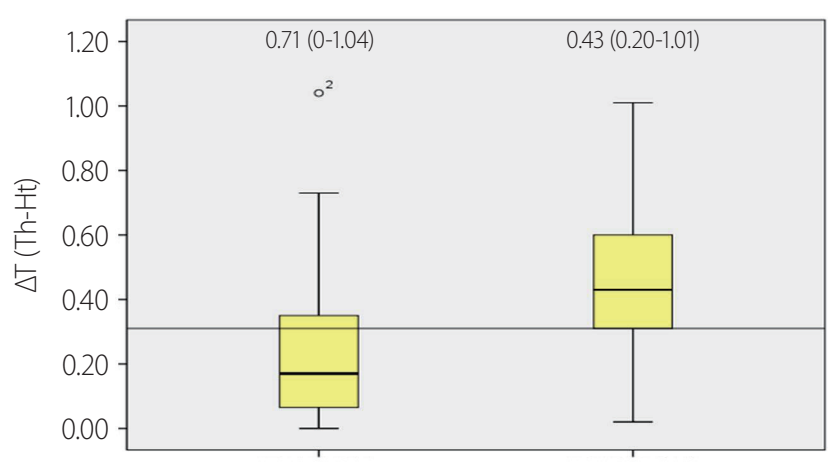

Class 1 (stage 1, 2) Class 2 (stage 3, 4, 5)

A

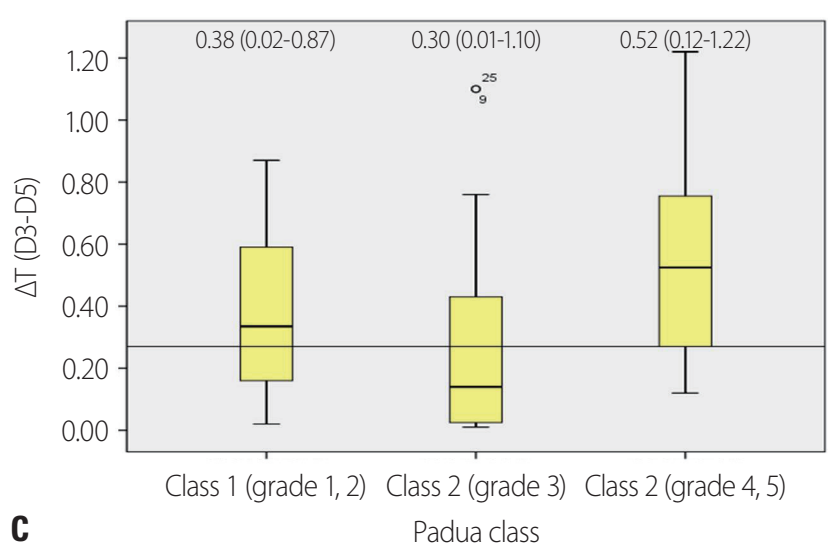

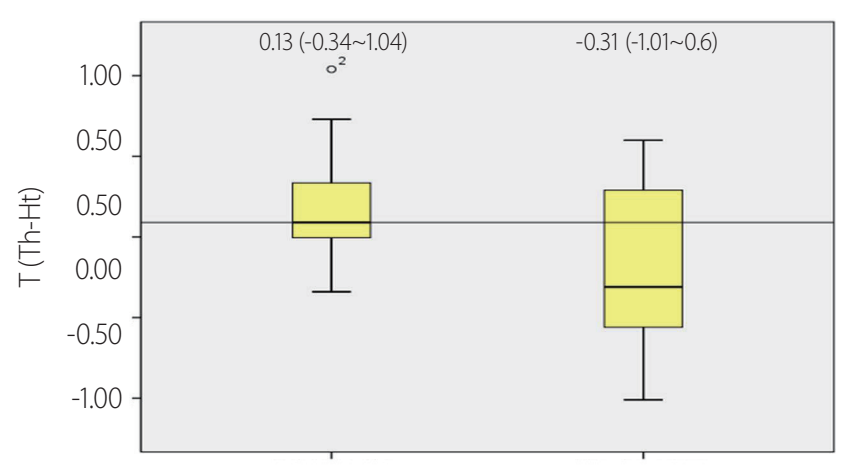

Class 1 (stage 1, 2) Class 2 (stage 3, 4, 5)

B $\mathrm{Hi}-\mathrm{Ob}$ class

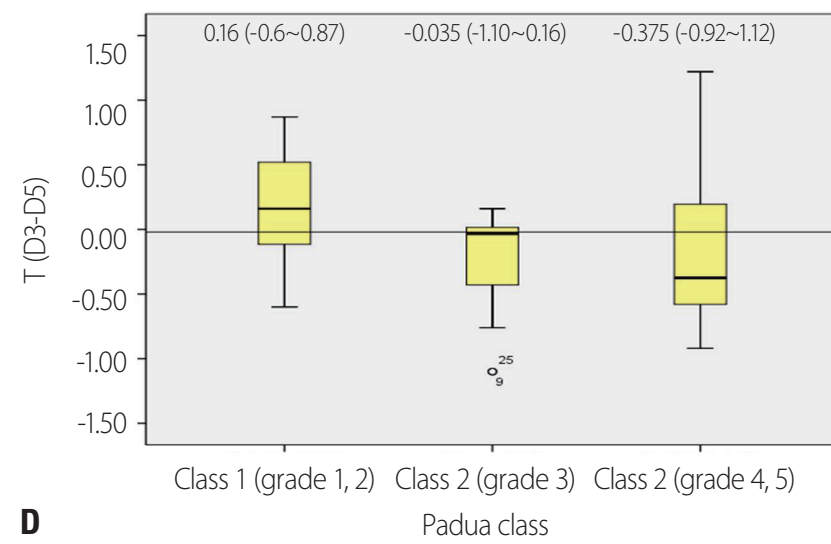

Fig. 2. (A) The mean and median values of the absolute temperature difference $(\Delta T)$ between the thenar $(T h)$ and hypothenar (Ht) regions in the same hand with carpal tunnel syndrome (CTS) were higher for class 2 on the historical-objective (Hi-Ob) scale. (B) The temperature was lower in Th (median nerve distribution) in hands with more-severe CTS. (C) The mean and median values of $\triangle T$ between digit 3 (D3) and digit 5 in the same hand with CTS were higher for class 3 on the Padua scale. (D) The temperature was lower for D3 (median nerve distribution) in hands with more-severe CTS. 
value of $1.0^{\circ} \mathrm{C}$ ) or NCS abnormalities resulted in a modest increase in the sensitivity from $91.4 \%$ to $94.3 \%$.

Regarding the 32 hands with CTS symptoms and NCSproven CTS, $\triangle T$ (Th-Ht) tended to be higher for class 2 on the Hi-Ob scale ( $p=0.085)$, while $\Delta T$ (D3-D5) tended to be higher for class 3 on the Padua scale $(p=0.106)$, which were considered more-advanced stages. Skin temperature was lower in hands with moderate-to-severe CTS than in hands with mild CTS (Fig. 2).

\section{Correlations between BCTQ, NCS, and IRT}

The SSS and FSS scores showed a strong mutual correlation (Spearman's rho, 0.882; $p<0.001$ ). However, they did not show a correlation with the Hi-Ob scale, Padua scale, and IRT parameters ( $\triangle T$ between $\mathrm{ROIs}, \mathrm{Ml}$, and $\mathrm{MU}$ ) in 32 hands with CTS symptoms and NCS-proven CTS. The average SSS and FSS scores did not differ significantly with the classes on the $\mathrm{Hi}$-Ob and Padua scales (Supplementary Fig. 1, 2). We further conducted a correlation analysis between SSS subscores concerning pain (items 1-5) and IRT parameters, which revealed no correlation. However, 11 hands with high SSS scores (> 4.0) showed a positive correlation with $\Delta \mathrm{T}(\mathrm{Th}-\mathrm{Ht})$ (Spearman's rho $=0.812 ; p=0.177$ ) after adjusting for age, sex, and BMI, and hands with CTS with higher SSS scores tended to be colder in the thenar region (Fig. 3).

We reviewed 11 hands with a discrepancy between

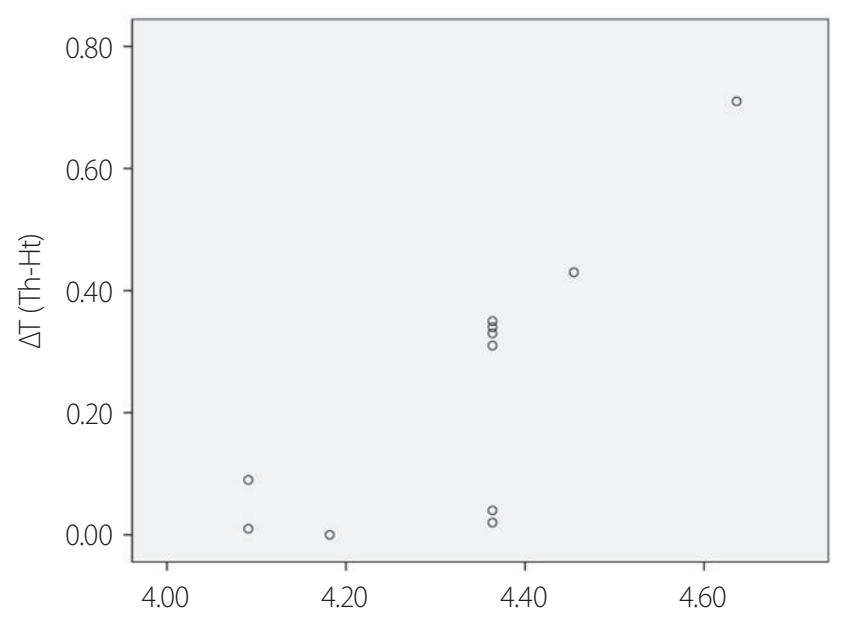

A

High SSS (> 4.0) symptoms and NCS results. The mean SSS and FSS scores for hands with CTS symptoms $(n=3)$ were comparably high, at 3.18 and 2.75 , respectively. The regional $\Delta T$ of the ROls in 11 hands did not show abnormal differences in the territory of the median nerve. However, the reliability of the interpretation was limited by the small number of samples available for inclusion in the statistical analysis.

\section{DISCUSSION}

Standard symptoms of CTS include paresthesia, dull and aching discomfort, weakness or clumsiness, dry skin, swelling, and color changes in the hands. ${ }^{10}$ Autonomic dysfunction might be accompanied by CTS more often than currently thought. Verghese et al. ${ }^{19}$ reported the presence of autonomic dysfunction in more than half of CTS cases. Some patients with CTS suffer from prominent pain along with paresthesia and numbness, suggesting the involvement of nociceptive fiber dysfunction, and hence small myelinated and unmyelinated nerve fibers in the pathogenesis of $\mathrm{CTS}^{20}$ Demyelination of large myelinated sensory nerve fibers occurs in the early stage of compressive neuropathy and causes tingling and numbness, which is why NCSs can detect CTS early and are widely used in clinical practice. Furthermore, small myelinated and unmyelinated $\mathrm{A}-\delta$ and

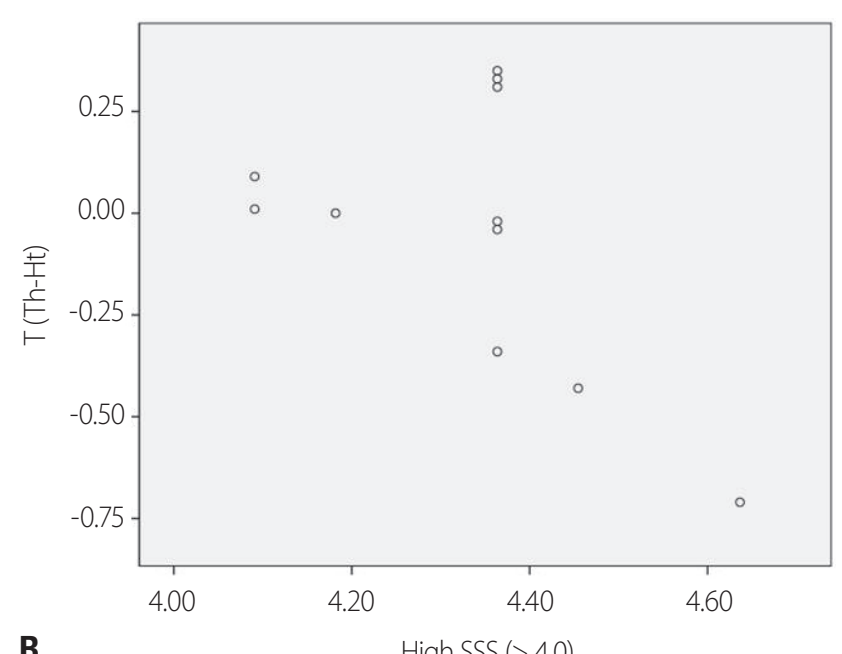

B

High SSS (> 4.0)

Fig. 3. (A) A positive correlation was observed between $\Delta T$ for the thenar ( $\mathrm{Th}$ ) and hypothenar ( $\mathrm{Ht}$ ) regions and the symptomatic severity score (of $>4.0$ ) in a hand with carpal tunnel syndrome (CTS). (B) The temperature was lower in Th (median nerve distribution) in hands with CTS with higher symptomatic severity score (SSS). 
C fibers are affected in the advanced stages of compression. However, some studies have suggested that distal degeneration of small myelinated sensory nerves and the loss of intraepidermal nerves are already seen in mild CTS or the early phase of the disease. ${ }^{21}$ Sympathetic nerve fibers have the same distribution as motor and sensory branches of peripheral nerves, which encase them. Moreover, these fibers are more common in the nerves that supply the hand and foot. $^{22}$

While NCS has been accepted as the gold standard for diagnosing CTS along with accurate clinical history-taking and examinations, it has inherent limitations in only evaluating the function of large myelinated fibers. ${ }^{6}$ This means that some patients with CTS exhibit normal NCS results, with Witt et al. ${ }^{2}$ reporting that this is the case in up to $25 \%$ of patients with CTS. This situation has resulted in some orthopedic surgeons considering NCSs unnecessary in patients with typical CTS. ${ }^{23}$ Several diagnostic tools are used to assess pain and small sensory nerve fiber function in CTS, including the sympathetic skin response, quantitative sensory testing, laser-evoked potentials, capillaroscopy, Doppler ultrasonography, skin biopsy, and IRT. 17,20,24-28

An IRT device detects infrared light emitted from the body (i.e., body heat) using a camera and displays this on a screen. It provides information about changes in cutaneous blood flow, which is regulated by sympathetic vasomotor nerve fibers. The advantages of IRT are that it is noninvasive, painless, and rapid, and shows physiological changes. IRT has been used to diagnose pain, inflammation, and cancerous diseases. ' In the neurological field, some studies have used IRT for discriminating neuropathic pain, such as nerve root irritation and compression, peripheral nerve injury, and entrapment, from nonneuropathic pain. However, studies using IRT for diagnosing CTS have produced conflicting results, such as 1) high sensitivity and specificity ${ }^{24,29}$ or 2) low sensitivity and/or low specificity. ${ }^{17,30}$

In this study, the side-to-side temperature differences in D2 were higher in patients with CTS than in healthy controls, and the median nerve distribution had higher temperature variability than had the ulnar distribution in hands with CTS, although the statistical significance of the difference decreased after adjusting for covariates. However, IRT did not have a high sensitivity when applied alone (although it did have a high specificity), and did not show an advantage when combined with NCS. This means that IRT cannot be implemented in place of or in addition to NCS in diagnosing CTS, which conforms with the results of previous studies. ${ }^{17,30}$ One particularly interesting finding was that lower temperatures in D3 and Th were observed in hands with moderate-to-severe CTS on physical examination and hands with severe CTS symptoms, which suggested sympathetic vasomotor dysregulation in hands with more-severe CTS.

There are several ways to evaluate the severity of CTS, including self-administered questionnaires, physician-oriented clinical scales, the degree of demyelination, and secondary axonal loss in NCSs. ${ }^{8,9,12}$ All of these approaches have been well validated, but they cannot predict treatment outcomes, and no correlation has been observed between the findings of these tools. ${ }^{2,31}$ The present study found a significant correlation between the SSS and FSS and between the Hi-Ob and Padua scales, but no correlation was observed between the scores for the self-administered questionnaires (i.e., SSS, FSS) and the objective measurement scales (i.e., the $\mathrm{Hi}-\mathrm{Ob}$ scale, Padua scale, and IRT parameters). These results suggest the need to develop other measurement tools that are strongly correlated with the objective status and evaluate the neuropathic pain aspect of CTS.

This study had several limitations. First, the numbers of enrolled patients and healthy controls were too small to obtain a high analytical power. Second, pain-specific questionnaires, such as the Neuropathic Pain Symptom Inventory or painDETECT, were not applied. Psychiatric surveys would help to quantify pain in patients with CTS. Third, including additional diagnostic tools to evaluate CTS would be better, especially regarding pain and small nerve fiber dysfunction. However, this study has strengths over previous studies in terms of focusing on the correlations between the symptom severity of patients, and objective examinations and diagnostic tests (NCS and IRT).

In conclusion, IRT is not advantageous over NCS in diagnosing and evaluating CTS. However, this study identified the presence of discrepancies between clinical diagnoses, subjective severity, and NCS results, and higher temperature variability in the median nerve distribution in hands with CTS with higher SSS scores and higher classes on the Hi-Ob scale. These observations mean that follow-up studies with a larger-scale and complementary design (e.g., selecting specific patient groups such as apparent painful clinical CTS 
with normal NCS findings) might be useful for clarifying whether IRT has practical usefulness in diagnosing CTS predominantly involving small sensory nerves, and in elucidating the associated relationships.

\section{Conflicts of Interest}

The authors declare no conflicts of interest relevant to this article.

\section{Funding}

This study was supported by the 2017 academic research funds of the Korean Society of Clinical Neurophysiology.

\section{Supplementary Material}

Supplementary Materials can be found with this article online https://doi.org/10.14253/acn.2021.23.2.99.

\section{REFERENCES}

1. Rempel D, Evanoff B, Amadio PC, de Krom M, Franklin G, Franzblau A, et al. Consensus criteria for the classification of carpal tunnel syndrome in epidemiologic studies. Am J Public Health 1998;88:1447-1451.

2. Witt JC, Hentz JG, Stevens JC. Carpal tunnel syndrome with normal nerve conduction studies. Muscle Nerve 2004;29:515-522.

3. Padua L, Padua R, Lo Monaco M, Aprile I, Tonali P. Multiperspective assessment of carpal tunnel syndrome: a multicenter study. Italian CTS Study Group. Neurology 1999;53:1654-1659.

4. de Campos CC, Manzano GM, Leopoldino JF, Nóbrega JA, Sañudo A, de Araujo Peres $C$, et al. The relationship between symptoms and electrophysiological detected compression of the median nerve at the wrist. Acta Neurol Scand 2004;1 10:398-402.

5. Chan L, Turner JA, Comstock BA, Levenson LM, Hollingworth W, Heagerty PJ, et al. The relationship between electrodiagnostic findings and patient symptoms and function in carpal tunnel syndrome. Arch Phys Med Rehabil 2007;88:19-24.

6. Werner RA, Andary M. Carpal tunnel syndrome: pathophysiology and clinical neurophysiology. Clin Neurophysiol 2002;113:13731381.

7. Nahm FS. Infrared thermography in pain medicine. Korean J Pain 2013;26:219-222

8. Levine DW, Simmons BP, Koris MJ, Daltroy LH, Hohl GG, Fossel $\mathrm{AH}$, et al. A self-administered questionnaire for the assessment of severity of symptoms and functional status in carpal tunnel syndrome. J Bone Joint Surg Am 1993;75:1585-1592.

9. Giannini F, Cioni R, Mondelli M, Padua R, Gregori B, D'Amico P, et al. A new clinical scale of carpal tunnel syndrome: validation of the measurement and clinical-neurophysiological assessment. Clin Neurophysiol 2002;113:71-77.

10. American Association of Electrodiagnostic Medicine, American Academy of Neurology, and American Academy of Physical Medicine and Rehabilitation. Practice parameter for electrodiagnostic studies in carpal tunnel syndrome: summary statement. Muscle Nerve 2002;25:918-922.

11. Werner RA, Andary M. Electrodiagnostic evaluation of carpal tunnel syndrome. Muscle Nerve 2011;44:597-607.

12. Padua L, LoMonaco M, Gregori B, Valente EM, Padua R, Tonali P. Neurophysiological classification and sensitivity in 500 carpal tunnel syndrome hands. Acta Neurol Scand 1997;96:211-217.

13. Campero M, Verdugo RJ, Ochoa JL. Vasomotor innervation of the skin of the hand: a contribution to the study of human anatomy. J Anat 1993;182(Pt 3):361-368.

14. Jones BF. A reappraisal of the use of infrared thermal image analysis in medicine. IEEE Trans Med Imaging 1998;17:1019-1027.

15. Park GY, Chun SI, Park Cl, Yim SY, Kim AY, Shin DB. Comparison of CT-myelography, electromyography and digital infrared thermographic imaging in lumbar herniated nucleus pulposus. J Korean Acad Rehab Med 1993;17:42-50.

16. Harper CM Jr, Low PA, Fealey RD, Chelimsky TC, Proper CJ, Gillen DA. Utility of thermography in the diagnosis of lumbosacral radiculopathy. Neurology 1991;41:1010-1014.

17. Meyers S, Cros D, Sherry B, Vermeire P. Liquid crystal thermography: quantitative studies of abnormalities in carpal tunnel syndrome. Neurology 1989;39:1465-1469.

18. Ming Z. Upper limb musculoskeletal disorders with special reference to sympathetic nerve functions and tactile sensation [dissertation]. [Kuopio]: University of Kuopio; 2007. 93.

19. Verghese J, Galanopoulou AS, Herskovitz S. Autonomic dysfunction in idiopathic carpal tunnel syndrome. Muscle Nerve 2000;23:1209-1213

20. Truini A, Padua L, Biasiotta A, Caliandro P, Pazzaglia C, Galeotti $F$, et al. Differential involvement of A-delta and A-beta fibres in neuropathic pain related to carpal tunnel syndrome. Pain 2009;145:105-109.

21. Schmid AB, Bland JD, Bhat MA, Bennett DL. The relationship of nerve fibre pathology to sensory function in entrapment neuropathy. Brain 2014;137(Pt 12):3186-3199. 
22. Aminoff MJ. Involvement of peripheral vasomotor fibres in carpal tunnel syndrome. J Neurol Neurosurg Psychiatry 1979;42:649655.

23. Lane LB, Starecki M, Olson A, Kohn N. Carpal tunnel syndrome diagnosis and treatment: a survey of members of the American Society For Surgery of the Hand. J Hand Surg Am 2014;39:21812187.e2184.

24. Ming Z, Zaproudina N, Siivola J, Nousiainen U, Pietikainen S. Sympathetic pathology evidenced by hand thermal anomalies in carpal tunnel syndrome. Pathophysiology 2005;12:137-141.

25. Mondelli M, Vecchiarelli B, Reale F, Marsili T, Giannini F. Sympathetic skin response before and after surgical release of carpal tunnel syndrome. Muscle Nerve 2001;24:130-133.

26. Goadsby PJ, Burke D. Deficits in the function of small and large afferent fibers in confirmed cases of carpal tunnel syndrome. Muscle Nerve 1994;17:614-622.
27. Zyluk A, Kosovets L. An assessment of the sympathetic function within the hand in patients with carpal tunnel syndrome. J Hand Surg Eur Vol 2010;35:402-408.

28. Clarke C, Christensen C, Curran MWT, Chan KM. Assessment of small sensory fiber function across the spectrum of severity in carpal tunnel syndrome patients. Muscle Nerve 2017;56:814-816.

29. Herrick RT, Herrick SK. Thermography in the detection of carpal tunnel syndrome and other compressive neuropathies. J Hand Surg Am 1987;12(5 Pt 2):943-949.

30. So YT, Olney RK, Aminoff MJ. Evaluation of thermography in the diagnosis of selected entrapment neuropathies. Neurology 1989;39:1-5.

31. Mondelli M, Reale F, Sicurelli F, Padua L. Relationship between the self-administered Boston questionnaire and electrophysiological findings in follow-up of surgically-treated carpal tunnel syndrome. J Hand Surg Br 2000;25:128-134. 